\title{
AVALIAÇÃO DA VARIÁVEL VISUAL TAMANHO APLICADA A MAPAS PERSPECTIVOS TRIDIMENSIONAIS
}

\author{
Evaluation of visual variable size applied to perspective tridimensional maps
}

\author{
Marcio Augusto Reolon Schmidt ${ }^{1}$ \\ Luciene Stamato Delazari ${ }^{2}$ \\ 1 Universidade Federal de Uberlândia. Programa de Pós-Graduação em Engenharia Civil. Faculdade de \\ Engenharia Civil. marcioschmidt@feciv.ufu.br
}

²Universidade Federal do Paraná. Programa de Pós-Graduação em Ciências Geodésicas. luciene@ufpr.br

\begin{abstract}
:
The adoption of a not orthogonal point of view in maps creation implies changes in the symbology design to make possible the cartographic communication. There are no general principles for 3D maps and misconceptions in the construction process of these maps may restrict or even impair the proper communication of cartographic features, and their spatial relationships represented. In addition, graphic design technologies allow quite complex and compelling visual solutions, but the problem of proper symbology is compounded by lack of knowledge of how users react to the this perspective representation and perceptual differences from conventional topographic map. Therefore, there is a need to adapt the cartographic representations to the $3 \mathrm{D}$ environment. This research proposes the evaluation of the visual variable size in some specific features on a 3D map and assesses its influence on the perception of expert users. To accomplish this, the research presents a test methodology that combines qualitative and quantitative techniques to measure the degree of success of the proposition. The results show different degrees of perception of visual variables in the visual differentiation process of landmarks.
\end{abstract}

Keywords: Map symbology, Relative size, Perception and cognition

\section{Resumo:}

A adoção de um ponto de vista não ortogonal na construção dos mapas implica em mudanças no processo construtivo da simbologia para que a comunicação cartográfica seja possível. Não existem princípios gerais para projetos cartográficos $3 \mathrm{D}$ e equívocos no processo de construção destes mapas podem restringir ou até mesmo impossibilitar a comunicação cartográfica adequada das feições representadas e suas relações espaciais. Além disso, as tecnologias de criação de imagens permitem soluções visuais bastante complexas e atrativas, mas o problema de uma simbologia adequada é agravado pela falta de conhecimento de como os usuários reagem à perspectiva da representação e às diferenças perceptivas em relação ao mapa topográfico convencional. Por isso existe a necessidade de adaptação das representações cartográficas para o ambiente 3D. Esta pesquisa propõe a avaliação da variável tamanho em algumas feições específicas em um mapa 3D e avalia a influência destas na percepção de usuários especialistas. Para alcançar este objetivo, a pesquisa apresenta uma metodologia de teste que combina técnicas 
qualitativas e quantitativas para mensurar o grau de sucesso da proposição. Os resultados apontam diferentes graus de percepção das variáveis visuais no processo de diferenciação de pontos de referência.

Palavras-chave: Simbologia de mapas 3D, Tamanho relativo, Percepção e cognição

\section{Introdução}

O termo "mapa 3D" tem sido amplamente utilizado na literatura científica como, por exemplo, por Terribilini, (1999), Darken e Paterson (2001), Haeberling (1999; 2002), Petrovic (2003), Harrower e Shessley (2005) e Davies e Peebles (2006). Mesmo que não haja consenso sobre a terminologia, estudos como o de Kraak (1988) afirma que uma representação cartográfica é considerada um mapa 3D se contém estímulos (depth cues) que fazem o usuário perceber o seu conteúdo como tridimensional. Para Haeberling (2002), a carta topográfica 3D é uma analogia de uma carta topográfica em papel, cuja descrição da área através da distribuição e características de representação das feições do terreno, é realizada tal qual ao mapa em papel, tais como a escala do mapa, mensurabilidade ou localização de objeto. O conceito de carta topográfica 3D não se afasta daquele de carta topográfica convencional senão pela inclusão da altura como atributo da terceira dimensão e da posição da câmera, a qual funciona como uma metáfora dos olhos do usuário. $\mathrm{O}$ maior impacto, neste caso, é a percepção do espaço numa vista limitada pela posição, atitude e configurações da câmera (perspectiva), os elementos presentes na interface e a simbologia em si.

De maneira análoga ao mapa convencional, o entendimento do uso de mapas 3D e o contexto do problema devem ser norteadores das decisões durante o estabelecimento da linguagem cartográfica e construção da simbologia. As tentativas de validação de uma linguagem cartográfica tridimensional de caráter geral por autores como Haeberling (1999, 2002), Terribilini (2001), Petrovic (2003) e Haeberling et al (2008) apontam que na vista perspectiva uma simbologia convencional como para mapas topográficos não é adequada, sendo necessário que a mesma seja tratada sob aspectos da cartografia temática. Por isso, o termo 3D é adequado desde que haja integração e apresentação da organização espacial do fenômeno na superfície do terreno de acordo com as convenções de simbolização e de generalização cartográfica, mesmo que o usuário perceba a paisagem representada como uma perspectiva tridimensional, embora esta seja representada na tela do dispositivo de visualização (computadores, celulares, tablets, entre outros) (Haeberling, 2002). Neste contexto, Petrovic (2003) afirma que as representações, ou modelos, tridimensionais só podem ser considerados mapas quando satisfazem algumas condições, semelhante ao que ocorre com mapa em papel, como ser projetados num sistema de coordenadas planas, de acordo com uma projeção cartográfica que resulta em deformações de valores conhecidos e acessíveis ao usuário; a informação apresentada ter sofrido generalização cartográfica; e o uso de uma linguagem cartográfica adequada para que haja comunicação entre o cartógrafo e o usuário do mapa. Surgem outros aspectos a serem considerados para o correto reconhecimento de símbolos tridimensionais como a percepção de profundidade (diferenças de escala), as condições atmosféricas, a visualização de azimute e ajustes de iluminação. Em mapas 3D é preciso analisar além dos aspectos técnicos e se concentrar nos aspectos mais sutis da representação que podem enriquecer a compreensão dos usuários. Elementos como a cor de fundo, ângulo de visada e abertura da câmera, e a própria distância entre os objetos numa cena, podem alterar a percepção de um símbolo. 
Esta pesquisa foca a variação da variável visual tamanho na construção de uma representação virtual e avalia o reconhecimento por parte de voluntários, com o objetivo de mensurar as alterações em função da mudança do ponto de vista e dos parâmetros de configuração da cena. Para isso é necessário entender como o mapa é percebido e como o conhecimento de percepção e cognição podem ser usados para permitir a comunicação cartográfica em mapas para navegação virtual não imersivos.

\section{Revisão bibliográfica}

Montelo (2002) afirma que o mapeamento cognitivo engloba os processos cognitivos que permitem às pessoas adquirir, codificar, armazenar, recuperar e manipular informações sobre a natureza do seu ambiente espacial. Estas informações são os atributos e posições relativas de pessoas e objetos no ambiente, e formam um componente essencial do processo adaptativo de tomada de decisão espacial. Para Lloyd e Bunch (2005a), mapas cognitivos são estruturas de memórias internas que representam a informação espacial aprendida. Ao interagir com o espaço geográfico, ou suas representações, o usuário organiza e atualiza as suas percepções e preferências dentro de uma matriz espacial. Essa mistura de informação qualitativa e espacial no mapa cognitivo permite que os indivíduos tomem decisões em um contexto espacial (Lobben, 2004).

A aplicação de mapas topográficos na navegação resulta na familiarização dos usuários com certas áreas geográficas, na medida em que se tornem capazes de planejar rotas e de se orientar no espaço (Lobben, 2004). Segundo a autora, o indivíduo inicia a navegação com um mapa cognitivo desenvolvido a partir de uma análise exploratória realizada com mapas, ou circulando pela área, e com a consulta repetida ao mapa durante a realização da tarefa de navegação a representação interna sobre a distribuição das feições é refinada.

Os mapas cognitivos são organizados segundo a estrutura da memória individual. Estes mapas são, portanto, rotulados e armazenados em uma superestrutura de onde podem ser acessados de acordo com a necessidade considerando a variação de escalas. Este mesmo fenômeno foi observado por Vinson (1999) que propõe que a simbologia de mapas considere esta variação de escalas.

O uso de mapas topográfico 3D para navegação apresenta outros problemas além daqueles de decodificação como no caso do mapa 2D. A maioria dos ambientes virtuais representa um espaço representado maior do que pode ser visto a partir de um único ponto de vista. Isto limita o número de feições apresentadas que podem ser selecionadas para ancorar o mapa cognitivo e os usuários, para navegar eficientemente dentro do ambiente, devem obter diferentes visões da cena para gerar um entendimento mais amplo da representação, através do movimento. Neste momento, a quantidade de detalhes e a forma como os símbolos foram construídos pode prejudicar ou facilitar a seleção de certas feições para serem usadas como referências espaciais.

Apesar de autores como Darken e Sibert (1993); Vinson (1999); Chittaro e Burigat (2004), salientarem que a restrição dos possíveis pontos de referência induzirem os usuários à erros de localização e à sensação de estarem perdidos durante a navegação, não se encontra na literatura princípios e diretrizes para comunicação de pontos de referência. As características individuais como a capacidade de rotação de objetos, identificação de símbolos e autolocalização e as de grupo como o treinamento formal, apontam Schmidt e Delazari (2013), contribuem para a aquisição de conhecimento espacial. De forma geral, Pinker (2009) teoriza que a disposição dos símbolos e suas relações com os símbolos nos seus entornos contribuem para a ancoragem da posição do usuário, 
afetam sua capacidade de autolocalização e permitem a passagem de um tipo de conhecimento para outro, ainda que as representações internas sejam diferentes para cada pessoa.

Entretanto, evidências coletadas em testes com usuários a vista em perspectiva aproximam a percepção do símbolo ao seu representante se o mesmo estimular o esboço primário da teoria de Marr (1982) (Schmidt 2012). Aliado a outras variáveis visuais que estimulem o contraste relativo podem contribuir para direcionar a atenção seletiva e contribuir que determinado símbolo seja selecionado como ponto de referência para ancoragem do mapa cognitivo. Estes símbolos diferenciam-se dos seus entornos para serem percebidas como tais em oposição ao que se torna o fundo da imagem. Este processo interativo é repetido indefinidamente a cada vez que se explora diferentes partes do mapa e a informação resultante é levada ao processamento cognitivo para associação de significado.

Se o agrupamento dos elementos básico visual for imediato, então a atenção seletiva favorece a identificação e reconhecimento de objetos e padrões. Este agrupamento pode ser realizado sob influência de diferentes fatores da Gestalt, de acordo com a disposição dos elementos na cena e, segundo seus atributos que contribuem para a heterogeneidade, influenciam a separação figurafundo. O resultado deste processo é a organização perceptiva da cena. A identificação de formas, cores e profundidade da cena tridimensional funciona como dados de entrada para os esquemas de conhecimento processarem a cena e participam da construção de uma representação interna que não é uma cópia cartográfica do mapa ou do mundo real, mas uma metáfora constituída de informações espaciais relevantes armazenadas.

Enquanto a Gestalt se preocupa com o fechamento dos símbolos e grupos, Reimer (2011) obteve resultados que indicam que o uso das variáveis visuais como tamanho e tom de cor destacam-se na formação de agrupamentos visuais de modo diferente dos produzidos pela Gestalt, mesmo quando estes símbolos estão bastante separados no mapa. Isto sugere um limiar para aplicação da Gestalt. Ou seja, ao observar um símbolo, o reconhecimento do mesmo se faz de modo integral, vendo o símbolo como um objeto completo e não as primitivas gráficas e variáveis visuais de maneira independente para em seguida organizar as relações entre objetos segundo a organização perceptiva. Dessa forma, pode-se afirmar que a Gestalt é mais voltada a formação dos objetos individuais enquanto que as variáveis visuais de Bertin permitem agrupamentos de símbolos distantes na representação.

A formação do esboço primário ocorre segundo os fatores, ou leis, da Gestalt para agrupamento (Maceachren, 1995, p 72): proximidade, semelhança, movimento comum, pregnância da forma (Pragnanzstufen), unicidade, continuidade, fechamento, simplicidade, experiência ou hábito, heterogeneidade, contorno, arredores (Surroundedness), orientação, e tamanho relativo. Este último, afirma que a menor de duas áreas tem maior probabilidade de ser percebida como figura. Este fator se aplica adequadamente a mapas com vista ortogonal para comparação de símbolos. Entretanto, nas cartas topográficas 3D, devido ao efeito da perspectiva, este fator pode não ser adequado. A partir do ponto de vista de quem navega existem sucessivos planos perpendiculares à linha de visada que podem sobrepor umas às outras formando uma imagem que transmite duas informações (figura 1). 


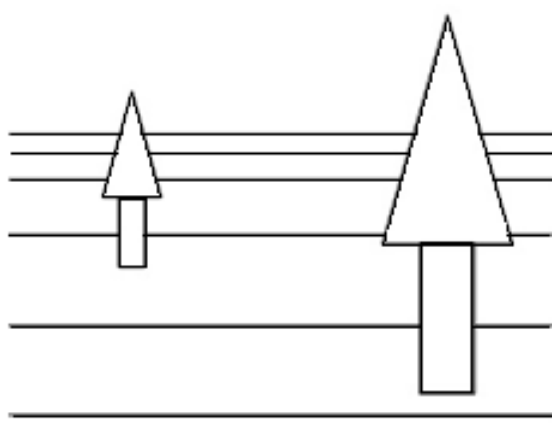

Figura 1: Símbolos de uma mesma classe de feição em posições diferentes em relação ao observador. Fonte: Autor

\section{Testes com Usuários}

\subsection{Criação do Mapa 3D}

Esta pesquisa utilizou uma combinação de dados obtidos da base cartográfica de 2007 do IPPUC (Instituto de Pesquisa e Planejamento Urbano de Curitiba) para a cidade de Curitiba, na escala 1:10.000, e a ocupação e uso do solo foi modificada com base na interpretação de aerofotos do município de Uberlândia (MG), na mesma escala, de 2010. A razão foi criar uma representação hibrida de modo a não ser reconhecida pelos voluntários e haver qualquer tipo de influência nas respostas dos testes. O projeto cartográfico considerou a classificação das feições segundo o mapeamento sistemático brasileiro na escala 1:50.000.

A proposição para construção dos símbolos seguiu a organização proposta por Haeberling (2002) composta de três passos: proposição geométrica, proposição visual e proposição de visualização. O primeiro passo pode ser entendido como a definição dos objetos em modo wireframe nos softwares CAD e sua distribuição na representação. A proposição visual trabalhou as variáveis visuais tamanho, tom e valor de cor, textura e padrão, orientação e com os aspectos gráficos especiais como a reflexão de superfícies. A proposição visual considerou os fatores de iluminação da cena, efeitos atmosféricos e ambientais, estrutura do céu (cor e esmaecimento), parâmetros de câmera como abertura e profundidade de campo, e nível de detalhamento. O processo é interativo, ou seja, cada passo da proposição pode ser reavaliado caso o resultado final não seja adequado à comunicação cartográfica, segundo a sensibilidade do cartógrafo.

Nesta pesquisa, a proposição geométrica do mapa base utilizou a variável tamanho para diferenciar, nas camadas de rodovias e vegetação, feições representativas das classes e, também, pontos de mudança de rota, como cruzamentos e curvas acentuadas. No primeiro caso, a razão foi estimular a percepção da hierarquia entre as rodovias representadas e, no segundo, foi o de destacar pontos comumente tomados como pontos de ancoragem.

A distribuição destes pontos atende a uma distribuição consistente de pontos de referência segundo uma matriz visual, na qual as relações espaciais entre os pontos possam ser relativamente determinadas, e cumpre com esta tarefa, pois favorece a seletividade dos símbolos. A distribuição dos pontos de referência foi realizada de modo que no mínimo dois pontos de referência sejam intervisíveis. A navegação pode ser facilitada se o usuário perceber que os objetos são identificados 
como pertencentes a uma mesma classe de elementos e distribuídos de modo que sua posição seja inequívoca no seu mapa cognitivo Segundo os indícios de necessidade de regularidade na distribuição espacial destacados por Pinker (2009), é importante destacar que os pontos de referência não tiveram suas posições e orientações alteradas, mas a sua distribuição na representação foi usada como critério para decidir se a feição seria ou não tomada como ponto de referência e, portanto, teria sua representação alterada pela variáveis visuais. $\mathrm{O}$ resultado dos mapas com a aplicação da variável visual é mostrado na Figura 2. As setas indicam aplicação da variável tamanho às estradas e às árvores.

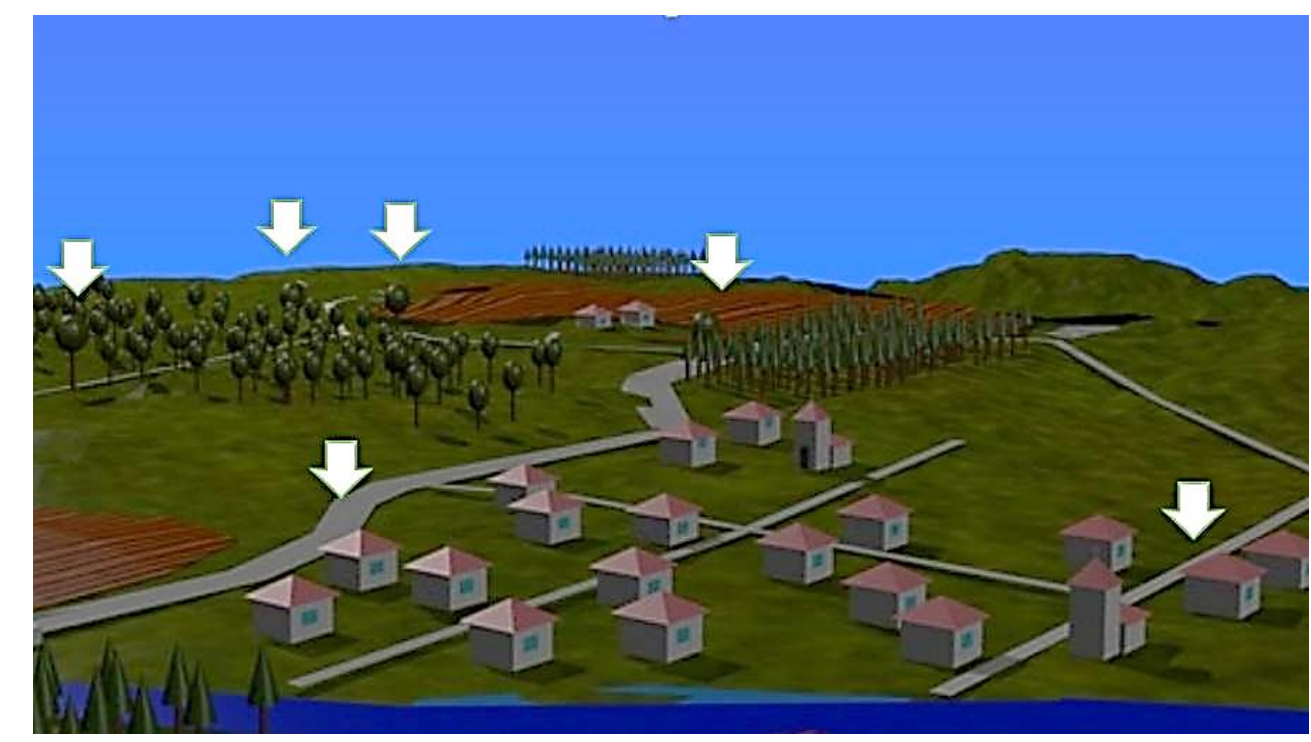

Figura 2: Aplicação da variável visual tamanho à representação padrão. Fonte: Autor

\subsection{Metodologia de teste}

O total de participantes foi de 43 voluntários sendo 22 da Universidade Federal do Paraná (UFPR) e 21 da Universidade Federal de Uberlândia (UFU). Os voluntários geraram 43 formulários com respectivos croquis que foram analisados segundo os critérios de número de pontos de referência e das diferenças das estimativas de posição, de distância e de orientação relativas.

A execução dos testes se iniciou com a identificação do perfil do usuário, através de perguntas de múltipla escolha que buscaram identificar o nível de instrução em cartografia do usuário, seus hábitos de navegação enquanto dirige ou caminha, tempo de formação, tempo que trabalho na área de produção de mapas, idade, entre outras. Essas perguntas tiveram o proposito identificar o grau de conhecimento dos participantes com a produção e uso de cartografia, além de aspectos individuais que poderiam afetar o entendimento da representação. Em seguida, foram realizados os testes com mapas.

O objetivo dos testes foi verificar a eficácia da variável visual tamanho na identificação dos símbolos nos quais esta variável foi aplicada, observando o contexto de separação figura-fundo, na formação de agrupamentos e classes de símbolos, para seleção dos pontos de referência e posterior formação de rotas. Para diminuir as variáveis de análise, tais como capacidade de interação com dispositivos digitais e de uso de ferramentas de navegação que não são pertinentes a esta pesquisa, os testes foram realizados com uso dos mapas topográficos $3 \mathrm{D}$ impressos. $\mathrm{O}$ 
voluntário deveria usar o mapa para responder a perguntas sobre a disposição e relacionamento espacial entre feições e, em seguida, elaborar os respectivos croquis. Por isso, foram usadas duas imagens. A primeira com simbologia padrão para todo o mapa 3D e a segunda com as feições próximas a pontos de mudança de rota e diferentes classes de importância das rodovias alteradas com uso da variável tamanho.

Inicialmente, a primeira imagem, com a simbologia padrão, foi apresentada ao usuário junto com a instrução: "Siga a estrada a partir da igreja mais ao norte e dirija-se ao replantio de pinheiros mais ao sul". O usuário produziu um croqui da primeira tarefa, e a imagem foi trocada pela segunda, com a simbologia modificada, e solicitou-se que ele executasse a mesma instrução para a produção de um novo croqui. Ao final foi questionado ao participante se ele(a) havia identificado ordem ou hierarquia em alguma classe perceptível nesta imagem. A estratégia adotada de comparar os croquis dos mapas 2D e 3D da mesma região permite inferir sobre a percepção do mapa. Por isso, ao final da sessão de testes um mapa topográfico em escala 1:50.000 foi apresentado e os voluntários desenharam mais uma vez um croqui. A função do uso deste mapa foi avaliar a capacidade do participante em ler um mapa com simbologia convencional em vista exocêntrica.

A partir dos elementos identificados nestes croquis foram calculadas as frequências das respostas dos voluntários segundo os critérios da quantidade de feições representadas, a recordação das suas características e a estimativa da posição relativa entre as feições. Posteriormente, estas informações e as frequências relativas foram analisadas para se determinar os graus de significância das respostas em relação à proposição desta pesquisa.

Do total de participantes foram selecionados aleatoriamente cinco participantes para a realização do teste Think Aloud. À medida que os usuários interagiam com os mapas, narravam suas ações e impressões em voz alta. As informações obtidas por este tipo de análise foram úteis para identificar quais são os pontos de referência utilizados no cumprimento da tarefa de planejamento da navegação e reconhecimento de feições de destaque, uma vez que ao desenhar o croqui os usuários lembram, processam e aplicam seus esquemas mentais na determinação da posição e das características das feições lembradas e, portanto, os pontos de referência e suas características não são necessariamente os mesmos desenhados.

\subsection{Resultados e discussões}

A variável tamanho foi utilizada na diferenciação das árvores próximas aos pontos de mudança de rota (curvas e cruzamentos de estradas) e na largura das estradas, porém mantendo os mesmos valores de cor da proposição original.

A tarefa neste teste era informar um caminho que partisse da igreja mais ao norte até o pinheiral que ficava mais ao sul (caminho de A até B). Na carta topográfica percebe-se quatro possíveis soluções para a tarefa. Como forma de atender as sugestões de Vinson (1999) e as discussões da proposição, as árvores mais próximas aos cruzamentos de vias foram diferenciadas pela variável tamanho aplicada à copa e altura em relação às árvores vizinhas. A diferenciação somente pela altura não é adequada neste caso, pois em uma atividade anterior à etapa dos testes foi verificado que as pessoas não percebem, ou subestimam, as variações somente em altura. Em razão disso, o raio das figuras geométricas que compõem as árvores foram alterados proporcionalmente à alteração em altura.

Os resultados mostram que, entre os 43 voluntários especialistas, 17 pessoas (40\%) informaram corretamente um dos caminhos possíveis e 26 pessoas $(60 \%)$ erraram a resposta devido a erros de 
orientação. Exemplos das respostas são apresentados na figura $3 a$ e $3 b$, respectivamente ao caminho errado e caminho possível.

Na figura 3a, o participante não identificou a orientação da câmera e, portanto, não conseguiu se orientar adequadamente. Como consequência a sua rota sai da área urbana e segue para o sentido contrário ao pretendido através da ponte. Porém, devido ao ponto de partida da rota desenhada no croqui, não se deve considerar a não percepção do pinheiral (solução do teste) ao fundo da cena como justificativa desta resposta. A rota informada neste croqui inicia-se na igreja mais ao sul e à esquerda da imagem. Nesta figura a igreja que está mais ao norte e à direita na imagem do teste não está representada. Considerando que na imagem de teste a disposição das duas é evidente, conclui-se que se trata de um erro de orientação.

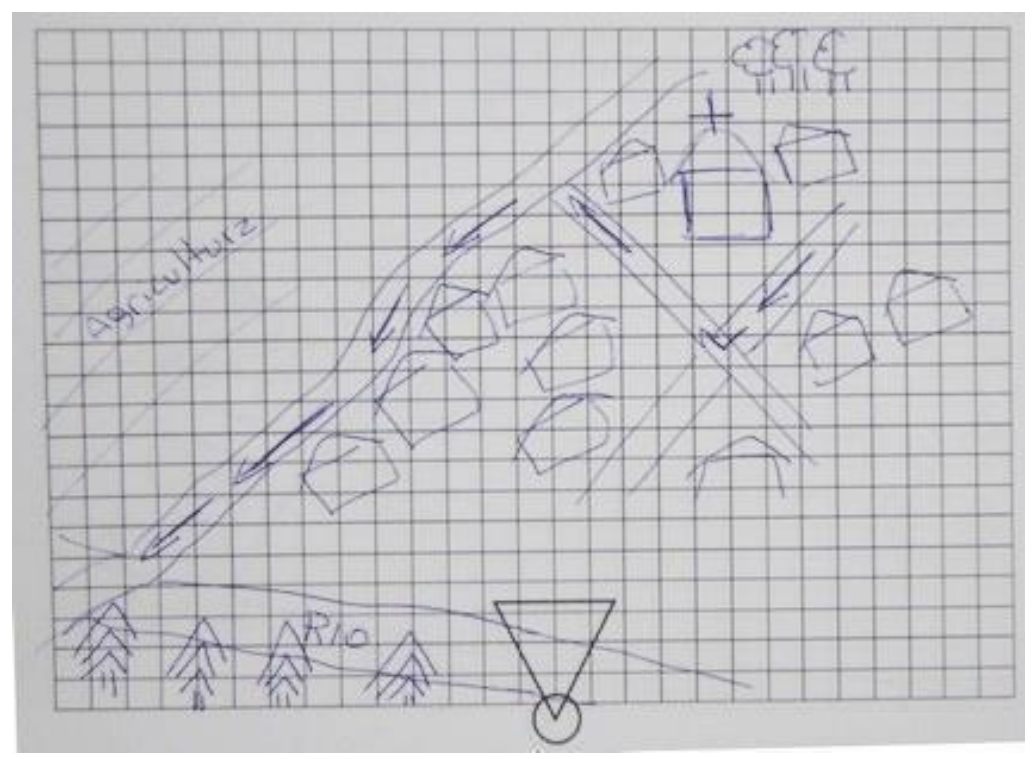

a)

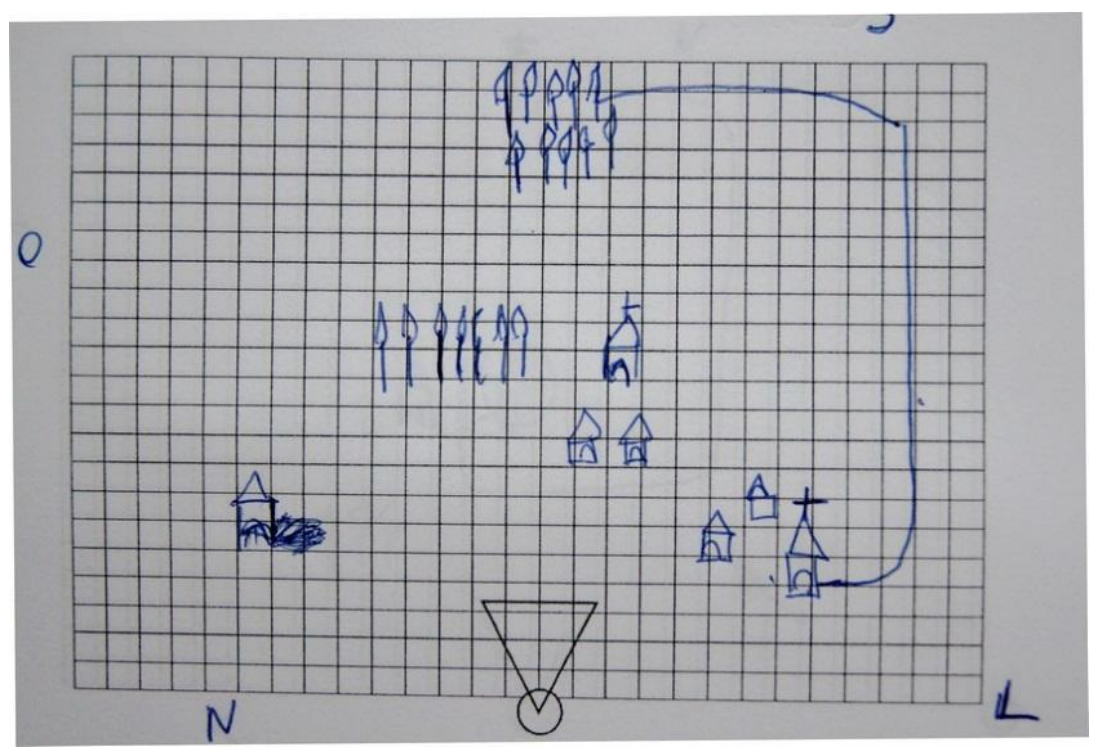

b)

Figura 3: a) croqui com erro de orientação e b) croqui com orientação correta. Fonte: autor 
$\mathrm{Na}$ figura 3b, o participante identifica a posição da sombra em relação à uma das casas representadas e informa a posição dos pontos cardeais. Neste croqui a posição relativa das igrejas na área urbana está claramente identificada e é indicado o caminho secundário que parte da igreja mais ao norte na carta topográfica convencional.

Apesar de 12 participantes (28\%) reconhecerem a variável visual tamanho, especialmente quando aplicada na representação das estradas, apenas três registros $(6,97 \%)$ foram coletados da aplicação desta variável às árvores. Uma justificativa é que o efeito perspectivo e as configurações da cena têm forte influência na percepção desta variável visual e consequentemente na hierarquia das classes, como as estradas. Isto demonstra a dificuldade em se usar esta variável visual em mapas 3D. Os resultados deste teste indicaram os piores resultados para a orientação geral dos croquis. Nos testes Think Aloud os participantes expressaram perceber poucas diferenças entre imagens e a identificação da variação em tamanho não foi reconhecida adequadamente.

\section{Conclusão}

Dos resultados obtidos é possível depreender que a variável tamanho sofre forte influência das configurações da câmera, em especial a atitude e os efeitos perspectivos associados ao ângulo de abertura. Além disso, os resultados sugerem que variações em altura e largura afastados do MDT são menos perceptíveis, como na representação das árvores, do que no nível do terreno, como no caso das estradas.

Outra conclusão refere-se a uma maior concentração de identificação das feições próximas a linha de visada do observador, neste teste coincidente com a linha de visada da câmera estática. Isso remete à discussão da figura 1 , ou seja, que o tamanho é percebido de forma mais adequada na comparação de objetos próximos e não de objetos em lados opostos da cena, por exemplo. Entretanto, essa afirmação pode estar relacionada aos efeitos perspectivos e merece aprofundamento.

Por fim, mais estudos são necessários para se estabelecer uma relação entre o exagero vertical a ser aplicado nos símbolos selecionados para sofrer a ação da variável tamanho de forma que o usuário tenha a sua atenção direcionada ao mesmo e essa feição possa ser utilizada como ponto de referência.

\section{AGRADECIMENTOS}

Ao CNPq pela bolsa parcial de doutorado e ao Edital $n^{\circ}$ 02/2012 MCTI/CNPq, processo ${ }^{\circ}$ 475169/2012-3, pelo financiamento parcial desta pesquisa ao primeiro autor. Ao Cnpq pela Bolsa Produtividade em Pesquisa, processo $n^{\circ}$ 30198/2014-4 e ao Edital Universal Chamada 2014 MCTI/CNPq processo $n^{\circ} 459300 / 2014-8$ ao segundo autor.

\section{REFERÊNCIAS}

Chittaro Luca, e Burigat Stefano. "3D Location-pointing as a Navigation Aid for Virtual Environments". Paper presented at the AVI 2004: 6th International Conference on Advanced Visual Interfaces. ACM Press, New York, 2004. 
Darken, Rudolph P., e Peterson, Barry. 2001. Spatial Orientation, Wayfinding, and Representation. Handbook of Virtual Environment Technology. Stanney, K. Ed.

Darken, Rudolph P, e Sibert, John. L. "A Toolset for Navigation in Virtual Environments". Paper presented at the 6th annual ACM Symposium on User Interface Software and Technology UIST'93. ACM 0.89791.628-X. Atlanta, Estados Unidos. 1993.

Davies, Clare., e Peebles, David. "Strategies for orientation: The role of 3D landmark salience and map alignment". In: D. McNamara e G. Trafton (Eds.). Paper presented at the Twenty-Ninth Annual Conference of the Cognitive Science Society. Mahwah, NJ. 2006.

Haeberling, Christian. "Symbolization in topographic 3D-maps: conceptual aspects for useroriented design". Paper presented at the 21th International Congress of Cartography. Otawa. V.2.1037-1044. 1999.

Haeberling, Christian. "3D-map presentation: A Systematic Evaluation of Important Graphic Aspects". Paper presented at the ICA Mountain Cartography Workshop "Mount Hood". International Cartographic Association. 2002.

Haeberling, Christian., Bär, Hans-Rudolf., Hurni, Lorenz. Proposed Cartographic Design Principles for 3D maps: A contribution to an Extended Cartographic Theory. Cartographica v. 43.n.3.(2008):175-188. Accessed August 02, 2009. Doi:10.3138/carto.43.3.175.

Harrower, Mark e Shessley, Benjamin C. 2005. "Moving Beyond Novelty: Creating Effective 3D Fly-Over Maps". Paper presented at the 22th International Cartographic Conference. La Coruña, Spain, July 9-16, 2005.

Kraak, Menno-Jan. "Computer-assisted cartographical three-dimensional imaging techniques". Delft University Press, Delft. Paper presented at the AutoCarto .1998.

Lloyd, Robert e., Bunch, Rick L. "Individual differences in map reading spatial abilities using perceptual and memory". Cartography and Geographic Information Science, 32 (2005): 33 -46. Accessed March 2012.

Lobben, Amy K. "Tasks, Strategies, and Cognitive Processes Associated With Navigational Map Reading: A Review Perspective". The Professional Geographer, 56(2) (2004): 270-281. Association of American Geographers. Blackwell Publishing. Accessed February 15, 2013.

Maceachren, Allan. 1995. How Maps Work: Representation, Visualization and Design. Ed. Guilford Press, Estados Unidos. ISBN 0-89862-589-0.

Montello Daniel R. Cognitive Map-Design Research in the Twentieth Century: Theoretical and Empirical Approaches. Cartography and Geographic Information Science. v 29, n 3. (2002): 283304. Accessed February 15, 2013.

Pinker, Steven. 2009. Como a mente funciona. Ed. Companhia das Letras. ISBN: 8571648468.

Reimer Andreas. "Squaring the circle: bivariate color maps and jacques bertin's concept of 'disassociation'”. Paper presented at the 25th International Cartographic Conference. Paris, França. ISBN: 978-1-907075-0-6. 2011 
Schmidt, Marcio Augusto Reolon e Delazari, Luciene Stamato. 2012. Avaliação de mapas topográficos 3D para navegação virtual. Boletim de Ciências Geodésicas, v. 18, no 4, (2012): $532-$ 548. Acessed Jan 28, 2013.

Terribilini, Andrea. "Maps in transition: development of interactive vector-based topographic 3Dmaps". Paper presented at the 19th International Cartographic Conference. Session 22-B. Ottawa. Canadá. 1999.

Vinson, Norman G. "Design Guidelines for Landmarks to Support Navigation in Virtual Environments". Paper presented at the CHI '99. Pittsburgh, USA. pp. 278-285. 1999

Submetido em Agosto de 2015.

Aceito em Outubro de 2015. 